\title{
Assessing discomfort after anaesthesia: should you ask the patient or read the record?
}

\author{
Marsha M Cohen, Peter G Duncan, Donald P DeBoer
}

\begin{abstract}
Objective-To assess the quality of anaesthesia care from the patients' viewpoint compared with the hospital record.

Design-Prospective study during 1988-9. Setting-Four teaching hospitals (A-D) in Canada.

Patients-15 960 inpatients receiving anaesthetic requiring at least an overnight stay, for whom an interview and review of hospital records within 72 hours of surgery were complete.
\end{abstract}

Main measures-Rates of postoperative symptoms of discomfort (nausea or vomiting, headache, back pain, sore throat, eye symptoms, and tingling) according to the hospital record versus interview and the relation between symptoms and patients' satisfaction with the anaesthetic experience.

Results-The preparation of completed interviews ranged from $31 \cdot 0 \%$ to $72 \cdot 7 \%$, owing mainly to patients discharge (hospitals A and B) and severity of illness (C and $D$ ). Interviewed patients were similar to all inpatients in the hospitals but were younger and healthier and more had had effective operations and were general surgical than cardiovascular or neurosurgical patients. In all, $26 \%$ to $46 \%$ of patients at the four hospitals reported at least one symptom of discomfort. Agreement between interviews and hospital records was low, symptoms being more commonly reported by interview than in the record (for example, headache was reported for $5 \cdot 8 \%-17 \%$ of patients compared with $0 \cdot 3 \%-3 \cdot 0 \%$ in hospital records). After controlling for case mix patients who reported at least one symptom were 2.91 times $(95 \%$ confidence interval $1 \cdot 89$ to $4 \cdot 50$ ) more likely to be dissatisfied with their anaesthetic care than patients who did not.

Conclusions-Anaesthesia services are typically neglected in studies of hospital quality, yet patients express considerable anxiety about anaesthetic care. Monitoring and recording patients' discomfort clearly need to be improved if the quality of anaesthesia is to be properly evaluated.

(Quality in Health Care 1994;3:137-141)

\section{Introduction}

Traditionally, quality of care in anaesthesia has been assessed by emphasising rates of death or major adverse events such as cardiac arrest, particularly among high risk patients undergoing cardiovascular or neurosurgical procedures. However, recent studies of modern anaesthesia care showed that among general surgical patients the contribution of anaesthesia to these major adverse events is very small. ${ }^{1}{ }^{2}$ We previously argued that major adverse events occur too infrequently to be used as a sole measure of quality of care in anaesthesia; alternative approaches are needed. ${ }^{2}{ }^{3}$ Fundamentally, the assessment of quality of care must be related to patients and what is of ultimate importance to them. Athough the prime concern of patients may be "making it through" the operation, their positive attitude when recalling anaesthesia can be compromised because of high rates of troublesome postoperative events such as nausea or vomiting, headache, back pain, and sore throat.

We carried out a large study in four Canadian teaching hospitals, in which information was collected on 37665 anaesthetics administered to adults. As part of the assessment, patients were interviewed in hospital after their operation about their anaesthetic experience.

In this report we focus on the following questions. Firstly, what are the rates of symptoms of discomfort among a general surgical population in the immediate postoperative period? Secondly, does the reporting of these adverse events differ with source of information - namely, the patient versus the hospital record? Thirdly, is the degree of satisfaction with the anaesthetic experience affected by the presence of symptoms of discomfort? Finally, what is the relation between the reporting of satisfaction or dissatisfaction and the recording of symptoms of discomfort in the hospital record?

\section{Methods}

After approval from institutional and university ethics committees had been received and during 12 months (1988-9) each adult, nonobstetric patient who received an anaesthetic at one of the four participating teaching hospitals was included in a standardised follow up study. Full details of the study protocol have been published, ${ }^{4}$ including the results for outpatients. ${ }^{5}$ Briefly, anaesthetists completed a modified anaesthesia record in a check off format which included information about the patient, the surgical procedure, and the anaesthetic. The preoperative health status of each patient was determined with the American Society of Anesthesiologists' 
physical status score ${ }^{6}$ which rates patients on a 1 to 5 scale from healthy (1) to not expected to survive the operation (5). This scoring system predicts mortality and major morbidity in the postoperative period. ${ }^{17}$ The surgical procedure or procedures were recorded and subsequently converted into the equivalent International Classification of Diseases (clinical modification) procedure code.

During the procedure the anaesthetist noted on the data collection form, the patient's course and therapeutic regimen as well as any adverse events that may have occurred. In the postanaesthetic care unit any untoward events were added to the same form by the unit's nursing staff.

Within 72 hours a research nurse visited inpatients and performed a standardised, structured interview. The items included in the interview were determined by a panel of clinical anaesthetists and an anaesthesia nurse (who had several years' experience in interviewing patients after their operation as part of an anaesthesia follow up programme). ${ }^{8}$ These items included nausea or vomiting, sore throat, headache, back pain, muscle pain, eye problems, or tingling or numbness. Although these factors may reflect care after surgery and are not always attributable to anaesthesia, they are of interest to anaesthetists because they are potentially modifiable or preventable. For example, nausea or vomiting may be caused by the anaesthetic administered (or how it is administered); sore throats by intubation, suctioning, or lack of humidity; and headache by spinal anaesthesia but also by drugs used or by fasting. Severe muscle pain may occur after ill positioning during surgery or if paralysing drugs are used. Back pain may be due to positioning during surgery, prolonged lack of movement, or poor hospital beds and tingling or numbness may be due to malpositioning of the limbs, poorly positioned intravenous drips, and, rarely, as a sequel to nerve blockade by a local anaesthetic technique.

The interview included closed format questions on the presence or absence (from the time since the operation) of these symptoms. The research nurses were instructed to use common terminology if the patient did not understand the question. If a symptom was present its severity was rated in two ways. Firstly, patients were asked if they had received therapy or treatment for that problem (drugs or other therapy) and, secondly, they were asked to rate the intensity of the symptom on a 5 point visual analogue scale ranging from 1 (mild) to 5 (excruciating). ${ }^{9}$ At the end of the interview, patients were asked if they were: "very satisfied, satisfied, somewhat satisfied, dissatisfied or very dissatisfied with their anaesthetic experience." If patients did not understand English, were too ill, or could not be contacted (after two attempts) the interview was not completed.

The research nurse also reviewed the patient's hospital record after the interview to determine the occurrence of adverse events as recorded by ward staff. The recording of symptoms in the ward will vary by recorder, ward, and hospital protocols. Our hospital record review included physicians' and nurses' notes and drug sheets. When there was no mention of a particular therapy in the medical or nurses' notes but a related therapy was recorded elsewhere (for example, physiotherapy), then this was also recorded.

The interview and hospital record review protocols were developed at one hospital and tested by two pairs of research nurses visiting the same 75 patients. The inter-rater agreements on the items tested were over $95 \%$. These protocols were then incorporated into the follow up visits of a further several hundred patients at one hospital before being extended to the other hospitals. The research nurses were trained by the project director, who visited each study site to ensure that the study protocols were appropriately instituted at each hospital and thereafter maintained fortnightly telephone contact with each centre to follow progress of the study and to ensure that the definitions and protocols were properly managed.

The proportion of patients who were interviewed and the reasons for not interviewing were determined for each hospital. Additionally, the proportion interviewed was determined according to age, sex, physical status score, emergency versus elective operation, and type of operation. The patients included in the statistical analysis were those for whom an interview and a hospital record review had been completed. Agreement between the patient and the hospital record was assessed using the $\kappa$ statistic $^{10}$ for the more prevalent symptoms of nausea or vomiting, headache, sore throat, and back pain. McNemar's test was used to compare the patients' responses with the data in the hospital record to determine which was superior in elucidating symptoms.

In this study three symptoms were emphasised: back pain, sore throat, and headache, and the analyses were performed with and without the dominating effect of nausea or vomiting. Rates of and risk factors for postoperative nausea or vomiting have been presented elsewhere, ${ }^{11}$ and, unlike the three other symptoms, nausea or vomiting has been well described and studied. ${ }^{12}$ With the patient interview as the source of the information, the rate of each symptom was determined (per 1000 anaesthetics) as well as the proportion of patients who reported the symptom as being severe (visual analogue scale score of 3 or more) or having been treated for that symptom. Dissatisfaction with anaesthesia was defined as being either dissatisfied or very dissatisfied.

The relation between having a symptom of postoperative discomfort and being dissatisfied was determined, firstly, for the three symptoms of headache, back pain, or sore throat (504 patients) and for these three symptoms plus nausea or vomiting (3497 patients), as elucidated by hospital record review, and also for the three or four symptoms using the interview as the source of the information. 
Table 1 Percentage (number) of inpatients interviewed, by hospital

\begin{tabular}{lcccc}
\hline & \multicolumn{4}{c}{ Hospital } \\
\cline { 2 - 5 } & $A$ & $B$ & $C$ & $D$ \\
\hline No of patients & 9305 & 6654 & 6095 & 5144 \\
With completed interview & $61 \cdot 7(5749)$ & $39 \cdot 0(2595)$ & $72 \cdot 8(4436)$ & $62 \cdot 4(3212)$ \\
Discharged early & $14 \cdot 8(1377)$ & $29 \cdot 1(1936)$ & $9 \cdot 7(591)$ & $6 \cdot 2(319)$ \\
Patient not found & $3 \cdot 8(354)$ & $3 \cdot 8(253)$ & $0 \cdot 4(24)$ & $0 \cdot 5(26)$ \\
Non-English speaking & $2 \cdot 6(242)$ & $1 \cdot 6(106)$ & $1 \cdot 0(61)$ & $0 \cdot 7(36)$ \\
Confused & $2 \cdot 2(205)$ & $2 \cdot 5(166)$ & $1 \cdot 9(116)$ & $1 \cdot 9(98)$ \\
Too sick for interview & $5 \cdot 8(540)$ & $10 \cdot 6(705)$ & $11 \cdot 1(677)$ & $22 \cdot 6(1162)$ \\
Refused & $0 \cdot 1(9)$ & $0 \cdot 1(7)$ & $0 \cdot 0(-)$ & $0 \cdot 0(-)$ \\
Not interviewed for other reason & $6 \cdot 6(614)$ & $10 \cdot 3(685)$ & $1 \cdot 9(116)$ & $4 \cdot 9(252)$ \\
With missing or miscoded records & $2 \cdot 3(215)$ & $3 \cdot 1(201)$ & $1 \cdot 2(74)$ & $0 \cdot 8(39)$ \\
\hline
\end{tabular}

To examine risk factors for dissatisfaction we used a multiple logistic regression and included various case $\mathrm{mix}$ and anaesthetic factors (age, sex, physical status score, elective versus emergency operation, duration of anaesthesia, use of inhalational technique, use of narcotics) as well as the hospital and whether or not any of the four symptoms had occurred. To determine statistical significance we calculated the $95 \%$ confidence intervals for each risk factor in the model. If the value of the relative odds ratio is greater than 1 (and the lower $95 \%$ confidence limit is also greater than 1) then that risk factor is significantly associated with an increased risk of dissatisfaction. If the relative odds is less than 1 (and the upper 95\% confidence limit is less than 1) then that factor is associated with a lower risk of dissatisfaction. Goodness of fit of the data to the logistic regression model was determined using the Hosmer-Lemeshow statistic. $^{13}$

\section{Results}

Among 27198 anaesthetics administered to inpatients (entailing at least an overnight stay in hospital), interviews were completed for 15992 patients and an interview and a hospital record review were completed for 15960 patients. The proportion of completed interviews ranged for $39.0 \%$ at hospital $\mathrm{B}$ to $72 \cdot 7 \%$ at hospital $\mathrm{C}$ (table 1 ). The reasons for not completing an interview differed among

Table 2 Representativeness of interviewed patients to all surgical patients treated at four study hospitals

\begin{tabular}{lcc}
\hline & $\begin{array}{c}\%(N o) \text { of all inpatients } \\
(n=27198)\end{array}$ & $\begin{array}{c}\%(\text { No) of interview sample } \\
(n=15977)\end{array}$ \\
\hline Age (years): & & \\
$<40$ & $33 \cdot 1(9003)$ & $33 \cdot 6(5368)$ \\
$40-59$ & $24 \cdot 8(6745)$ & $26 \cdot 7(4266)$ \\
$60-79$ & $34 \cdot 7(9437)$ & $34 \cdot 3(5480)$ \\
$\geqslant 80$ & $7 \cdot 4(2013)$ & $5 \cdot 4(863)$ \\
Sex: & & \\
Female & $51 \cdot 0(13871)$ & $49 \cdot 9(7973)$ \\
Male & $49 \cdot 0(13327)$ & $50 \cdot 1(8004)$ \\
Physical status score: & & \\
1 & $27 \cdot 3(7425)$ & $30 \cdot 5(4873)$ \\
2 & $40 \cdot 1(10906)$ & $44 \cdot 9(7174)$ \\
3 & $25 \cdot 0(6800)$ & $22 \cdot 3(3563)$ \\
4 and 5 & $7 \cdot 6(2067)$ & $2 \cdot 3(367)$ \\
Emergency or elective operation: & & \\
Emergency & $15 \cdot 8(4297)$ & $13 \cdot 5(2157)$ \\
Elective & $84 \cdot 2(22901)$ & $86 \cdot 5(13820)$ \\
Type of surgery: & & \\
Cardiac or neurosurgery & $10 \cdot 4(2829)$ & $5 \cdot 1(815)$ \\
Respiratory & $2 \cdot 4(653)$ & $2 \cdot 2(351)$ \\
Digestive & $15 \cdot 9(4325)$ & $19 \cdot 0(3036)$ \\
Gynaecological & $9 \cdot 6(2611)$ & $9 \cdot 4(1502)$ \\
Musculoskeletal & $21 \cdot 8(5929)$ & $26 \cdot 2(4186)$ \\
All other & $39 \cdot 9(10851)$ & $28 \cdot 1(6087)$ \\
\hline
\end{tabular}

Goodness of fit $\chi^{2}$ test significant at the $p=0.0001$ for all variables. the four hospitals: for hospitals A and B the main reason was the high number of patients discharged early (that is, staying only one night) and for hospitals $C$ and $D$, the research nurses judged that many patients were too ill to be interviewed. There were few patients who refused to be interviewed $(0 \cdot 1 \%$ or less).

Overall, the interviewed patients were very similar to all inpatients at the four hospitals (table 2). The interviewed sample was somewhat younger and healthier than the entire patient population. However, more interviewed patients had had elective operations and were general surgical patients than cardiovascular or neurosurgical patients.

The percentage of patients who on interview reported headache ranged from $5.8 \%$ at hospital C to $17 \cdot 0 \%$ at hospital B (table 3). Conversely, the proportion of hospital records in which a headache was noted varied from $0.3 \%$ at hospital D to $3.0 \%$ at hospital B. The proportion of concurrent negative responses for both interview and the hospital record ranged from $82.2 \%$ to $93.2 \%$ whereas that of concurrent positive answers from both sources varied from $0 \cdot 2 \%$ to $2 \cdot 2 \%$. One per cent of hospital records or less noted a headache denied by the patient; however, a significant proportion of patients (up to $14.8 \%$ ) claimed a headache that was not recorded in the hospital record. The overall agreement between the interview and the hospital record was poor, with $\kappa$ values ranging from 0.02 to 0.31 .

Agreement between the interview and the hospital record was even less for sore throat and back pain. $\kappa$ values for sore throat across the four hospitals ranged from 0.03 to 0.07 and for back pain from $0 \cdot 1$ to $0 \cdot 17$. $\kappa$ values for nausea or vomiting were somewhat improved, ranging from $0 \cdot 19$ at hospital $D$ to 0.60 at hospital A. Symptoms were much more commonly reported by patients than recorded in the hospital record, and this was seen at each hospital (McNemar's test, $\mathrm{p}<0.0001)$. The recording of these symptoms was slightly higher at hospitals $B$ and $C$ than at hospitals $\mathrm{A}$ and $\mathrm{D}$.

The percentage of interviewed patients reporting symptoms ranged considerably across the four hospitals, as did the percentage who claimed that the symptom was severe (as measured by the visual analogue scale or by the proportion claiming to have had treatment) (table 4). The rate of such symptoms as eye problems or numbness or tingling were very infrequent. However, the proportion of patients who had at least one symptom was relatively high, ranging from $26 \%$ at hospital A to $45.8 \%$ at hospital $\mathrm{B}$. The percentage of patients who claimed dissatisfaction with their anaesthesia care was low, and this varied from 0.2 to $1.7 \%$ across the four hospitals.

Patients who reported a discomfort symptom were 2.91 times as likely to be dissatisfied with their anaesthesia care as patients who did not (table 5). Case mix characteristics such as age, sex, and physical status scores did not predict dissatisfaction with anaesthesia care nor did duration of anaesthesia or use of narcotics. Patients at hospital D were much 
Table 3 Reporting of headache on patient interview versus review of hospital records, by hospital

\begin{tabular}{|c|c|c|c|c|}
\hline & \multicolumn{2}{|c|}{ Interview } & \multirow[t]{2}{*}{ Total } & \multirow[t]{2}{*}{$\kappa$} \\
\hline & Not reported & Reported & & \\
\hline \multicolumn{5}{|c|}{ Hospital $A$} \\
\hline $\begin{array}{l}\text { Review: } \\
\text { Not reported } \\
\text { Reported }\end{array}$ & $\begin{array}{c}5173(90 \cdot 6) \\
45(0 \cdot 8)\end{array}$ & $\begin{array}{r}453(7 \cdot 9) \\
37(0 \cdot 6)\end{array}$ & $\begin{array}{c}5626(98 \cdot 6) \\
82(1 \cdot 4)\end{array}$ & $0 \cdot 11$ \\
\hline Total & $5218(91 \cdot 4)$ & $490(8 \cdot 6)$ & & \\
\hline \multicolumn{5}{|c|}{ Hospital B } \\
\hline $\begin{array}{l}\text { Review: } \\
\text { Not reported } \\
\text { Reported }\end{array}$ & $\begin{array}{c}2133(82 \cdot 2) \\
21(0 \cdot 8)\end{array}$ & $\begin{array}{c}383(14 \cdot 8) \\
58(2 \cdot 2)\end{array}$ & $\begin{array}{c}2516(97 \cdot 0) \\
79(3 \cdot 0)\end{array}$ & $0 \cdot 18$ \\
\hline Total & $2154(83 \cdot 0)$ & $441(17 \cdot 0)$ & & \\
\hline \multicolumn{5}{|c|}{ Hospital C } \\
\hline $\begin{array}{l}\text { Review: } \\
\text { Not reported } \\
\text { Reported }\end{array}$ & $\begin{array}{c}4145(93 \cdot 2) \\
43(1 \cdot 0)\end{array}$ & $\begin{array}{r}196(4 \cdot 4) \\
60(1 \cdot 4)\end{array}$ & $\begin{array}{c}4341(97 \cdot 7) \\
103(2 \cdot 3)\end{array}$ & $0 \cdot 31$ \\
\hline Total & $4188(94 \cdot 2)$ & $256(5 \cdot 8)$ & & \\
\hline \multicolumn{5}{|c|}{ Hospital D } \\
\hline $\begin{array}{l}\text { Review: } \\
\text { Not reported } \\
\text { Reported }\end{array}$ & $\begin{array}{c}2893(90 \cdot 0) \\
6(0 \cdot 2)\end{array}$ & $\begin{array}{r}309(9 \cdot 6) \\
5(0 \cdot 2)\end{array}$ & $\begin{array}{c}3202(99 \cdot 7) \\
11(0 \cdot 3)\end{array}$ & 0.02 \\
\hline Total & $2899(90 \cdot 2)$ & $314(9 \cdot 8)$ & & \\
\hline
\end{tabular}

Table 4 Rate of symptoms per 1000 anaesthetics reported on interview

\begin{tabular}{lcccc}
\hline & \multicolumn{5}{c}{ Hospital } \\
\cline { 2 - 5 } & $A$ & $B$ & $C$ & $D$ \\
\hline No of patients interviewed & 5749 & 2595 & 4436 & 3212 \\
\% (No) of patients with: & & & & \\
Sore throat & $12 \cdot 3(707)$ & $20 \cdot 4(529)$ & $19 \cdot 8(878)$ & $24 \cdot 1(774)$ \\
$\quad$ Severe & $24 \cdot 5(173)$ & $22 \cdot 9(121)$ & $20 \cdot 8(183)$ & $71 \cdot 6(554)$ \\
$\quad$ Treated & $20 \cdot 7(146)$ & $13 \cdot 8(73)$ & $7 \cdot 9(69)$ & $5 \cdot 7(44)$ \\
Headache & $8 \cdot 7(499)$ & $17 \cdot 0(441)$ & $5 \cdot 8(257)$ & $9 \cdot 8(315)$ \\
Severe & $42 \cdot 3(211)$ & $18 \cdot 4(81)$ & $27 \cdot 6(71)$ & $62 \cdot 5(197)$ \\
Treated & $55 \cdot 3(276)$ & $51 \cdot 7(228)$ & $47 \cdot 1(121)$ & $33 \cdot 9(107)$ \\
Back pain & $9 \cdot 5(546)$ & $9 \cdot 1(236)$ & $1 \cdot 7(75)$ & $2 \cdot 2(71)$ \\
Severe & $44 \cdot 7(244)$ & $37 \cdot 7(89)$ & $40 \cdot 0(30)$ & $78 \cdot 9(56)$ \\
Treated & $46 \cdot 9(256)$ & $63 \cdot 1(149)$ & $48 \cdot 0(36)$ & $43 \cdot 7(31)$ \\
Muscle pain & $3 \cdot 3(190)$ & $9 \cdot 4(243)$ & $2 \cdot 8(124)$ & $4 \cdot 1(132)$ \\
Severe & $48 \cdot 9(93)$ & $23 \cdot 0(56)$ & $19 \cdot 4(24)$ & $53 \cdot 0(70)$ \\
Treated & $40 \cdot 0(76)$ & $57 \cdot 2(139)$ & $10 \cdot 5(13)$ & $17 \cdot 4(23)$ \\
Eye problem & $0 \cdot 6(35)$ & $2 \cdot 6(67)$ & $0 \cdot 1(4)$ & $1 \cdot 0(32)$ \\
Tingling & $0 \cdot 3(17)$ & $0 \cdot 1(3)$ & $0 \cdot 2(9)$ & $1 \cdot 3(42)$ \\
Excessive drowsiness ${ }^{\star}$ & $2 \cdot 1(120)$ & $1 \cdot 3(34)$ & Not recorded & $3 \cdot 1(100)$ \\
At least one symptom & $26 \cdot 8(1541)$ & $45 \cdot 8(1189)$ & $27 \cdot 0(1198)$ & $34 \cdot 7(1115)$ \\
Dissatisfaction & $0 \cdot 7(40)$ & $1 \cdot 0(26)$ & $0 \cdot 2(9)$ & $1 \cdot 7(54)$ \\
\hline
\end{tabular}

${ }^{\star}$ Recorded by observation, not interview. more likely to be dissatisfied (relative odds ratio 2.26) compared with those at hospital A and patients at hospital $\mathrm{C}$ were much less dissatisfied (relative odds 0.44).

\section{Discussion}

This study is one of the very few to assess the patient's view of common symptoms of postoperative discomfort. None the less, it has several limitations. Firstly, at hospital B a lower proportion of patients was interviewed relative to that in the other hospitals so a bias may have been introduced. Hospital B had only a half time nurse researcher, and it was not possible to interview all eligible patients. However, the profile of patients interviewed at hospital B was not different from that of patients at the other hospitals, and data were presented by hospital for most of the analyses. Secondly, several circumstances affecting the data collected were beyond the control of the investigator - for example, patients receiving drugs for surgical pain or receiving prophylactic anti-emetics would be less likely to notice symptoms of discomfort. Thirdly, we did not record the number and types of treatments for various symptoms, and patients' recall of treatment may not be reliable. Also we did not assess various factors such as preoperative education, expectations, or cultural factors which might have affected patients' expression of their symptoms or satisfaction.

Moerman $e t$ al asked 658 patients attending an outpatient clinic what they remembered about their previous anaesthetic. ${ }^{14}$ Most recollections related to the postoperative period, and $21 \%$ of those interviewed recalled nausea or vomiting and drowsiness. Of 250 patients asked to rate their satisfaction with their previous anaesthetic, about a quarter rated it as bad or very bad. The authors found no relation between experiencing nausea or vomiting and being dissatisfied. Few details about the interview were given and an unstructured questionnaire seems to have been used. In Australia a postoperative interview using a structured instrument was carried

Table 5 Factors associated with dissatisfaction

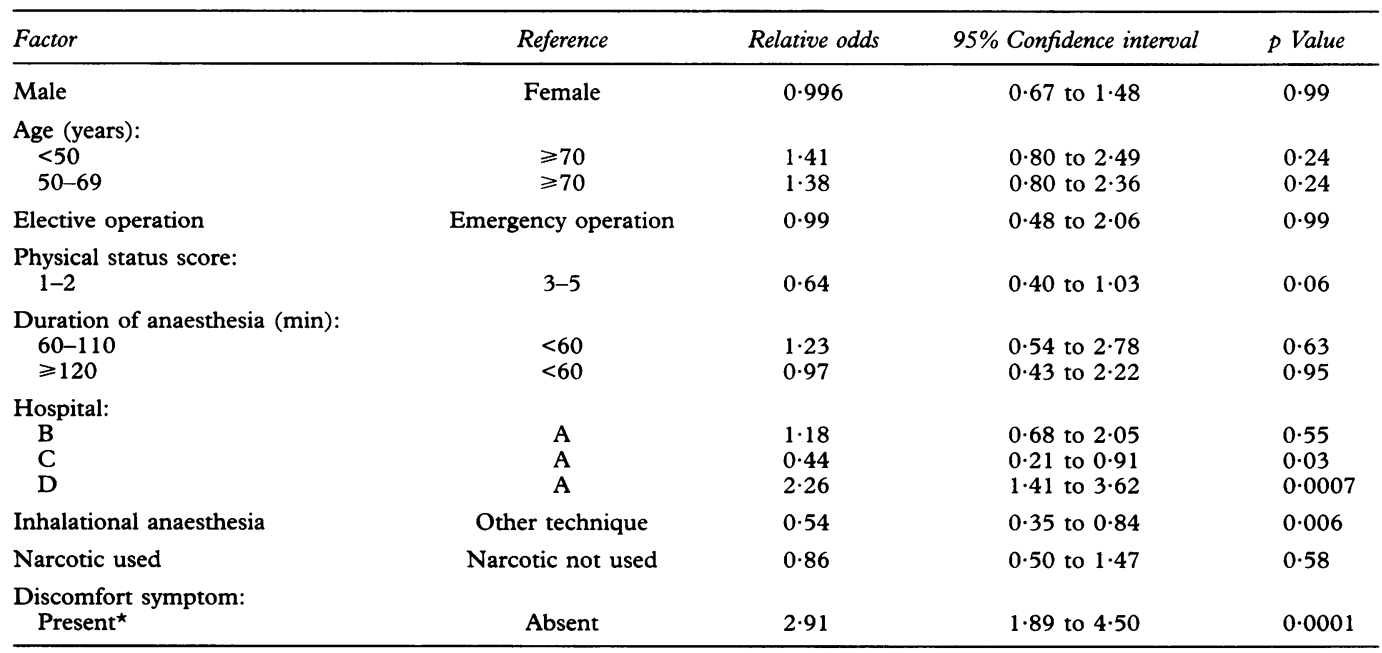

*At least one symptom of headache, sore throat, back pain, nausea or vomiting.

Hosmer-Lemeshow statistic 13.35, $\mathrm{p}=0 \cdot 1003$. 
out by a nurse for 125 patients. ${ }^{15}$ Satisfaction with anaesthesia was very high but several complaints such as sore throat, nausea or vomiting, muscle pain, shivering, and headache were found. A study of 129 patients in the Netherlands found similar postoperative complaints but, it did not assess satisfaction or quality of the anaesthetic. ${ }^{16}$

Few studies have compared the results of direct interviews with those obtained from hospital or other medical records. Some studies compared patients' recall of use of drugs ${ }^{17}$ or self reported illnesses. ${ }^{18-21}$ However, we are unaware of any recent studies which compared postoperative symptoms claimed by patients with those recorded in hospital records.

The symptoms experienced by patients were seldom reflected in the hospital record, and this may have several explanations. Charting protocols may vary among nursing staff, even across wards within the same hospital, and the importance of recording symptoms of discomfort differs among nursing staff. Only unusual events may be noted, since some staff expect that all patients undergo these problems after surgery and thus these events are not routinely recorded. Nursing staff to patient ratios may be inadequate to provide awareness of patients' discomfort. We cannot determine from our study whether the nonreporting of such symptoms reflected care received. Treatments may have been offered to patients with such symptoms, but if these were not pharmaceutical or physiotherapy treatments (for example, ice packs) they woud not have been recorded. Alternatively, anaesthetic, surgical, and nursing staff may accord little importance to these types of symptoms and this may be reflected in recording practices.

Undoubtedly, the patient's view needs more attention. When surveyed, patients wanted more information about their anaesthetic and a visit from the anaesthetist. ${ }^{22}$ Patients may expect unpleasantness after surgery and do not always want to bother busy nurses or doctors with what they may perceive as trivial complaints. None the less, monitoring and recording of symptoms of discomfort clearly needs to be improved if the quality of care in anaesthesiology is to be evaluated. In addition, better methods of assessing quality of care from patients' perspectives are needed. For example, a recent study successfully used a postal questionnaire to determine minor sequelae persisting after discharge from hospital. ${ }^{23}$

Anaesthesia services are typically neglected in studies of quality of hospital care and are not included in hospital databases, in mortality reviews, or in patient satisfaction surveys. Yet in the few surveys that have been done patients expressed considerable anxiety about anaesthetic care. ${ }^{24}$ Our study showed that patients were much more likely to be dissatisfied if they have had discomfort after surgery. Creative new approaches to address such concerns are needed.

We gratefully acknowledge the participation of the departments of anaesthesia at the four study hospitals and Drs W D B Pope, R Merchant, W A Tweed, and D Biehl. Comments on earlier drafts were provided by Dr David Naylor, Dr Hilary LlewellynThomas, and Elaine Thiel. This work was supported by a grant from the National Health Research Development Program, Health Canada, and by a National Health Scholar Award
(Health Canada) to MMC. Part of this research was presented at the Society for Medical Decision Making, October 1991, at the Society for Medic
Rochester, New York.

1 Cohen MM, Duncan PG, Tate RB. Does anesthesia contribute to operative mortality? $f A M A$ 1988;260: 2859-63.

2 Cohen MM, Duncan PG, Pope WDB, Biehl D, Tweed WA, MacWilliam $L$, et al. The Canadian four centre study of anaesthetic outcomes. II. Can outcomes
be used to assess the quality of anaesthesia care? Can $\mathcal{F}$ Anaesth 1992;39:430-9.

3 Orkin FK, Cohen MM, Duncan PG. The quest for meaningful outcomes. Anesthesiology 1993;78:417-22.

4 Cohen MM, Duncan PG, Tweed WA, Biehl D, Pope WDB, Perry M, et al. The Canadian four centre study of anaesthetic outcomes. I. Description of methods and populations. Can $\mathcal{F}$ Anaesth 1992;39:420-9.

5 Duncan PG, Cohen MM, Tweed WA, Biehl D, Pope WDB, Merchant RN, et al. The Canadian four centre study of anaesthetic outcomes. III. Are anaesthetic complications predictable in day surgical practice? Can $\mathcal{F}$ Anaesth 1992;39:440-8.

6 Saklad M. Grading of patients for surgical procedures. Anesthesiology 1941;2:281-5.

7 Cohen MM, Duncan PG. Physical status score and trends in anesthetic complications. $\mathcal{F}$ Clin Epidemiol 1988;41: 83-90.

8 Cohen MM, Duncan PG. Postoperative follow-up and quality of care. Seminars in Anesthesia 1988;7:270-7.

9 Cohen MM, Tate RB. Using the McGill pain questionnaire to study common postoperative complications. Pain 1989;39:275-9.

10 Fleiss JL. Statistical methods for rates and proportions. New York: J Wiley, 1981.

11 Cohen MM, Duncan PG, DeBoer DP, Tweed WA. The postoperative interview: assessing risk factors for nausea and vomiting. Anesth Analg 1994;78:7-16.

12 Lerman J. Surgical and patient factors involved in postoperative nausea and vomiting. Br f Anaesth 1992; 69(suppl 1):24-32S.

13 Hosmer DW, Lemeshow S. Applied logistic regression. New York: J Wiley, 1989.

14 Moerman N, van Dam FSAM, Oosting J. Recollection of general anaesthesia: a survey of anaesthesiological practice. Acta Anaesthesiol Scand 1992;36:767-71.

15 Dodds CP, Harding MI, More DG. Anaesthesia in an Australian private hospital: the consumer's view. Anaesth Intensive Care 1985;13:325-9.

16 van Wijk MGF, Smalhout B. A postoperative analysis of the patient's view of anaesthesia in a Netherlands' the patients

17 Paganini-Hill A, Ross RK. Reliability of recall of drug usage and other health related information. Am $\mathcal{f}$ Epidemiol 1982;116:114-22.

18 Harlow SD, Linet MS. Agreement between questionnaire data and medical records: the evidence for accuracy of recall. Am $\mathcal{F}$ Epidemiol 1994;129:233-48.

19 Colditz GA, Martin P, Stampfer MJ, Willett WC, Sampson L, Rosner B, et al. Validation of questionnaire information on risk factors and disease outcomes in a prospective cohort study of women. Am 7 Epidemiol 1986;123:894-900.

20 Bush TL, Miller SR, Golden AL, Hale WE. Self report and medical record report agreement of selected medical medical record report agreement of selected medical conditions

21 Linet MS, Harlow SD, McLaughlin JK, McCaffrey LD. A comparison of interview data and medical records for previous medical conditions and surgery. $\mathcal{F}$ Clin Epidemiol 1989;42:1207-13.

22 Wisiak UV, Kroll W, List W. Communication during the preoperative visit. Eur f Anaesthesiol 1991;8:65-8.

23 Philip BK. Patients' assessment of ambulatory anesthesia and surgery. $\mathcal{F}$ Clin Anesth 1992;4:355-8.

24 Shevde K, Panagopoulos G. A survey of 800 patients' knowledge, attitudes, and concerns regarding anesthesia. Anesth Analg 1991;73:190-8. 